\title{
Molecular cloning and characterization of a phospholipid hydroperoxide glutathione peroxidase gene from a blood fluke Schistosoma japonicum ${ }^{\text {is }}$
}

\author{
Ying Zhang ${ }^{\mathrm{a}}$, Yuan $\mathrm{He}^{\mathrm{b}}$, Li He ${ }^{\mathrm{b}}$, Hong-Ying Zong ${ }^{\mathrm{b}}$, Guo-Bin Cai ${ }^{\mathrm{b}, *}$ \\ ${ }^{a}$ Department of Medical Genetics, Wuhan University School of Basic Medicial Sciences,
} Wuhan, China

${ }^{\mathrm{b}}$ Department of Medical Parasitology, Wuhan University School of Basic Medicial Sciences, Wuhan, China

${ }^{2}$ Note: Nucleotide sequence data reported from this study are available in the GenBank database under accession number KJ941009.

* Corresponding author at: Department of Medical Parasitology, Wuhan University School of Basic Medicial Sciences, Wuhan 430071, China.

Tel.: +862768759143.

E-mail address: gbcai2011@whu.edu.cn (G.B. Cai) 


\section{ABSTRACT}

Phospholipid hydroperoxide glutathione peroxidase (PHGPx) is a major antioxidant enzyme and plays critical roles in the protection of cells against oxidative stress by catalysing reduction of lipid hydroperoxides. A full-length cDNA sequence corresponding to GPx gene from Schistosoma japonicum (designated SjGPx) was isolated and characterized. SjGPX contained an in-frame TGA codon for selenocysteine (Sec) and a concurrent Sec insertion sequence in its $3^{\prime}$-untranslated region. Protein encoded by $S j G P x$ demonstrated a primary structure characteristic to the PHGPx family, including preservation of catalytic domains and absence of the subunit interaction domains. Phylogenetic analysis revealed that the SjGPx was highly related to the other PHGPx-related members, and clustered into the trematode subclade II. Semi-quantitative reverse transcription PCR and western blotting showed that the SjGPx was mainly expressed in the female adults and eggs. RNA interference was employed to investigate the effects of knockdown of SjGPx. SjGPx expression level was significantly reduced on the 5th day post-RNAi. We observed a 53.86\% reduction in total GPx activity and the eggs severely deformed. Oxidative stimulation of viable worms with $\mathrm{H}_{2} \mathrm{O}_{2}$ or paraquat resulted in 1.6- to 2.1-fold induction of the GPx activity. Our results revealed that the SjGPx protein is selenium-dependent PHGPx, which might actively participate in the detoxification of oxidative damage during egg production.

Keywords: Phospholipid hydroperoxide glutathione peroxidase (GPx); Selenium-dependent GPx; RNA interference; Oxidative stress; Schistosoma japonicum 


\section{Introduction}

Schistosomiasis, a parasitic disease caused by Schistosoma, is a serious health problem in the tropical and subtropical regions. Schistosomiasis japonica is the most serious endemic zoonosis in China, with more than 0.32 million schistosomiasis patients across the country [1]. The pathogen, Schistosoma japonicum, was thought to be the most virulent species among the six schistosomes that could infect humans because of its high egg production and the over 40 species of wild and domesticated animals suspected to serve as reservoir hosts [2], which makes the transmission and epidemiological processes of this disease complex and difficult to control. Adult worms of $S$. japonicum settle in the mesenteric veins via the hepatic portal system. Most eggs lodge in the venules of the intestine, others are carried retrograde by the portal venous system to the liver, in which they become trapped in the portal triad [3]. During its migration through the tissues of the definitive host, the parasite might be continuously exposed to oxidative stresses, from reactive oxygen species (ROS, such as $\mathrm{O}_{2}{ }^{-}, \mathrm{H}_{2} \mathrm{O}_{2}, \mathrm{OH}^{*}$, ${ }_{1} \mathrm{O}_{2}$, and lipid hydroperoxides) generated by endogenous metabolism, host inflammatory and immune cells, and other oxidative pathways [4]. ROS play pivotal roles not only in control of cellular redox homeostasis but also in induction of cellular proliferation or apoptosis by activating signaling molecules [5]. However, the unbalanced generation of ROS may cause chemical damage to the cells, which might induce breakage of DNA strands, protein oxidation, polysaccharide depolymerization, membrane-lipid peroxidation and impairment of signal transduction from membrane receptors in various physiological processes [6]. In order to overcome these stressful conditions and to maintain its life-span, the fluke has evolved a series of multi-layered enzymatic and non-enzymatic defence systems, which can remove the 
aggressive ROS and/or repair ROS-mediated cell damage [7]. Distinct antioxidant enzymatic activities such as catalase, superoxide dismutase (SOD), glutathione peroxidase (GPx), peroxiredoxin $(\mathrm{PRx})$ have been well characterized from numerous taxa. Trematode parasites are known to lack catalase, suggesting that GPx proteins play important antioxidant roles alone or together with other proteins [8-9].

GPx encompasses six distinct families of multiple isoenzymes, including GPx1 (classical/cytosolic/cellular GPx), GPx2 (gastrointestinal GPx), GPx3 (plasma GPx), GPx4 (phospholipid hydroperoxide GPx, PHGPx; E.C. 1.11.1.12), GPx5 (epididymis-specific GPx) and GPx6 (odourant metabolizing GPx), which catalyse the reduction of $\mathrm{H}_{2} \mathrm{O}_{2}$, organic hydroperoxides and lipid hydroperoxides by using glutathione as a reducing agent. The GPx families share similar structural and enzymatic properties with one another [10]. PHGPx proteins have many distinct features compared to the other GPx families, which perform functions in a monomeric form because they lack the subunit interaction domains [10-12]. The PHGPx molecules directly reduce hydroperoxidized phospholipids integrated into membranes [13]. Members of the other GPx families, however, interfere with lipid peroxidation only via a concerted operation with phospholipase, which implies that PHGPxs are deeply associated with the repair of disrupted biomembranes [14]. GPxs might also constitute the front line of enzymatic defence to ensure their survival against host immune cell-derived ROS in parasitic helminthes, which cause chronic infections [15] The parasitic helminth GPx families show a certain degree of biased distribution across taxa. GPx proteins homologous to mammalian GPx3 have been characterized in the filarial nematodes including Brugia pahangi, Dirofilaria immitis and Wuchereria bancrofti [16]; while information on the 
PHGPx (GPx4) members is obtainable from trematodes such as Schistosoma mansoni [17-19], Clonorchis sinensis [20] and Paragonimus westermani [21] Observations regarding the tissue distribution of the $C$. sinensis, $P$. westermani and $S$. mansoni PHGPxs were shown to be largely localized in vitellocytes within vitelline follicles and eggs [20-22].

RNA interference (RNAi), which results in post-transcriptional gene silencing in a wide variety of organisms [23], was used in human parasitic worms, including schistosomes [24-27]. In eukaryotic cells, double-stranded RNA (dsRNA) can be recognized by the RNA induced silencing complex which effects degradation of the target mRNA, and abolishes expression of the corresponding protein [28]. dsRNAs, which can be synthesized in large quantities and transfected into target cells in the presence of lipofectamine reagents [29], are the most commonly used reagents for RNAi in cultured parasites [24,30].

In this study, we isolated and characterized a novel PHGPx gene (SjGPx) from the blood fluke $S$. japonicum. Molecular characteristics of $S j G P x$ were described, including selenocysteine insertion sequence (SECIS) elements, amino acid primary structures, and phylogenetic positions among various members of GPx families. The native SjGPx was analyzed by semi-quantitative reverse transcription PCR (RT-PCR), and western blotting. We employed a lipofectamine transfection procedure to introduce dsRNAs into cultured schistosome, and investigated the inhibitory effects of dsRNAs-mediated gene knockdown of SjGPx on intrauterine egg formation in S. japonicum. Finally, we employed a in vitro experiment to demonstrated that oxidative stress-inducible chemicals such as paraquat, $\mathrm{H}_{2} \mathrm{O}_{2}$ substantially augmented the expression of SjGPx in viable worms. 


\section{Materials and methods}

\subsection{Parasite}

Oncomelania hupensis snails infected with S. japonicum (Chinese mainland strain) were collected from an epidemic area of schistosomiasis in Hunan Province, China. Specific pathogen-free (SPF) BALB/c mice (female, 10 weeks of age), which were obtained from Wuhan University Laboratory Animal Center, were infected percutaneously through shaven abdomen with 20 cercariae of $S$. japonicum using an established method [31]. The mice were euthanized on day 42 after infection, male and female adult worms were recovered by the perfusion of hepatic portal veins, meantime, and the eggs were harvested from the livers of infected mice by comminution and layered-filtration with nylon mesh [32]. All samples were washed extensively to remove host cells or debris. Eventually, all the samples were either frozen and kept in liquid nitrogen, or immediately used for nucleic acid extraction. The use of animals was approved by the Animal Ethics Committee of Wuhan University.

\subsection{Isolation of S. japonicum cDNA encoding SjGPX}

A non-redundant GenBank database was screened with the nucleotide sequence of $S$. mansoni GPx (AY729668) used as query in the BLAST searches at National Center for Biotechnology Information (NCBI, http://www.ncbi.nlm.njh.gov). A clone (CV689936) isolated during the BLAST searches were used as query for the more comprehensive GPx sequence information from EST and genomic databases of S. japonicum (Shanghai Center for Life Science and Biotechnology Information, http://lifecenter.sgst.cn/sj.do). A total of forty-one entries were selected and two contigs were obtained by using a CAP3 Assembly Program (http://seq.cs.iastate.edu/cap3.html). A S. japonicum cDNA library (currently 
available in our laboratory) was screened using the DNA fragment of one contig as a probe. The cDNA from positive clone, designated SjGPx, was amplified by PCR using T3 and T7 promoter primers, cloned into pGEM-T easy vector (Takara, Shiga, Japan) and sequenced to obtain a full-length cDNA. The deduced amino acid (aa) sequence of $S j G P x$ was determined using the Open Reading Frame (ORF) Finder program at NCBI (http://www.ncbi.nlm.nih.gov/gorf/gorf.html). The selenocysteine (Sec) insertion sequence (SECIS) was detected using the SECISearch program (ver2.19;

http://genome.unl.edu/SECISearch.html). The Compute pI/Mw program (http://www.expasy.ch/tools/pi_tool.html) was performed for the calculation of the theoretical molecular weight $(\mathrm{Mr})$ and isoelectric point $(\mathrm{pI})$ value. The putative $\mathrm{N}$-terminal hydrophobic signal peptide was predicted by using the SignalP program (http://www.cbs.dtu.dk/services/SignalP).

\subsection{Amino acid sequence alignment and Phylogenetic analysis}

The translated aa sequence of SjGPx was used as query in a series of BLAST searches, to retrieve the closely matched sequences from a variety of public databases. Forty-eight reference sequences were retrieved, according to their homogy values and the taxonomical distributions of their donors. Amino acid sequences were aligned using ClustalX and optimized with GeneDoc (http://www.psc.edu./biomed/genedoc) programs, respectively. A phylogenetic analysis was conducted by the neighbor-joining or maximum parsimony algorithm within the PHYLIP package (ver3.6b). Alignment gaps were removed as missing data and trees were displayed with TreeView. The statistical significance of each branching 
point was evaluated with 100 random samplings of the initial input using SEQBOOT software.

\subsection{RNA isolation, cDNA synthesis, and semi-quantitative RT-PCR}

42-day live adult worms were chilled on ice for several minutes to facilitate separation of mated pairs. Expression levels of $S j G P x$ transcript in parasite various developmental stages, including egg, cercariae, male and female adults, were determined by semi-quantitative RT-PCR. Total RNA was extracted from each of the stages using Trizol reagents (Gibco, Carlsbad, CA). The RNA samples were treated with RNase-free DNase (GIBCO BRL, Rockvile, MD, USA). Poly(A) ${ }^{+}$RNAs were prepared from the total RNAs by oligo(dT)-affinity chromatography (Qiagen, Valencia, CA). The first strand cDNAs were synthesized from $1 \mu \mathrm{g}$ of poly(A) ${ }^{+}$RNA and oligo-dT primers by using a RNA PCR Kit (AMV) Ver. 3.0 (TaKaRa, Shiga, Japan) under the manufacturer's instruction. The samples were then PCR-amplified using SjGPx specific primers (forward primer: 5'-GATGATTGATTGTTTCACTCGAG-3'; reverse primer: 5'-CTATTTACTGGAAACGTCAAGC-3') and the cDNAs as a template. The PCR was conducted with a thermal cycling profile of $94{ }^{\circ} \mathrm{C}$ for $4 \mathrm{~min}, 28$ cycles of $40 \mathrm{sec}$ at $94{ }^{\circ} \mathrm{C}, 45$ sec at $59{ }^{\circ} \mathrm{C}, 1 \mathrm{~min}$ at $72{ }^{\circ} \mathrm{C}$ and a final extension of $10 \mathrm{~min}$ at $72{ }^{\circ} \mathrm{C}$. Reaction with a primer pair for the S. japonicum alpha-tubulin gene (AY815746, SjTubulin-F, 5'-CTACTGTAGTGGATGAAGTGCGAAC-3' and SjTubulin-R, 5'-CAGCTGAAATTACTGGTGCATAAG-3') was utilized as an internal quantity control, and the absence of any contaminating chromosomal DNA was verified via the preparation of 
reactions without reverse transcriptase during the first round of cDNA synthesis. The reaction products were resolved by $2 \%$ agarose gel electrophoresis and stained by ethidium bromide staining and detected by UV transillumination.

\subsection{Site-directed mutagenesis, expression and purification of recombinant SjGPx protein}

The gene segments spanning the SjGPx ORF region were amplified by PCR using cDNA as a template, as described above, and then cloned into pGEM T-Easy vector (Promega). The unusual Sec codon (TGA) was converted into a sense Cys codon (TGC), by using a complementary primer pair (5'-CTACTGAGTGCGGTCTCGCAGGCACAAACTATC-3' and 5'-GATAGTTTGTGCCTGCGAGACCGCACTCAGTAG-3') and the QuikChange Site-Directed Mutagenesis Kit (Stratagene, La Jolla, CA, USA). Competent Escherichia coli DH5 $\alpha$ cells were transformed with the mutated plasmid and the nucleotide sequence was verified by sequencing. Nucleotide corresponding to the mature domain of $S j G P x$ was amplified from the positive plasmid using specific primers containing EcoR I and Xho I sites (forward: 5'-CCGAATTCGGTCTCGCAGGCACAAACTATC-3'; reverse: 5'-GGCTCGAGCTATTTACTGGAAACGTCAAGC-3'). The PCR product was purified with a QIAquick PCR Purification Kit (Qiagen, Valencia, CA, USA), and then cloned into the pET-28a (+) vector (Novagen, Madison, WI, USA). E. coli DH5 $\alpha$ cells were then transformed with the restructured plasmids, and the accuracy of the nucleotide sequences was verified by sequencing. E. coli BL21 (DE3) cells were introduced the plasmid DNA exhibiting the correct codons. Expression of the recombinant $\mathrm{SjGPx}(\mathrm{rSjGPx})$ protein was induced by treating the transformant with $0.5 \mathrm{mM}$ isopropyl- $\beta$-D-thiogalactopyranoside (IPTG) at $37^{\circ} \mathrm{C}$. The bacterial cells were sonicated and the rSjGPx proteins were purified by Ni-NTA agarose chromatography (Amersham Biosciences). The eluted proteins were examined by $12 \%$ SDS-PAGE, and the purified rSjGPx protein was used to generate specific antibody. 


\subsection{Generation of specific polyclonal antibody and Western blotting}

Six-week-old, SPF female BALB/c mice were immunized subcutaneously with the purified rSjGPx proteins (30 $\mu$ g each) in Freund's complete adjuvant (Sigma, USA).

Subsequent injections using the equal amounts of the protein in Freund's incomplete adjuvant (Sigma, USA) were followed for two times at 2-week intervals. After 2 more weeks, the mice received intravenously boosters with the proteins $(10 \mu \mathrm{g})$. Seven days after the final booster, the mice were euthanised and blood was collected by heart puncture. The blood was centrifuged for $10 \mathrm{~min}$ at $3,000 \mathrm{~g}$ at $4{ }^{\circ} \mathrm{C}$ and the antisera were stored at $-80{ }^{\circ} \mathrm{C}$ until use.

The egg, cercariae and adult worms of the parasite were ground in a Teflon pestle-homogenizer in phosphate buffered saline (PBS, $140 \mathrm{mM} \mathrm{NaCl}, 2.7 \mathrm{mM} \mathrm{KCl}, 10 \mathrm{mM}$ $\mathrm{Na}_{2} \mathrm{HPO}_{4}$ and $1.8 \mathrm{mM} \mathrm{KH}_{2} \mathrm{PO}_{4} ; \mathrm{pH}$ 7.2) containing $2 \%$ SDS and a protease inhibitor cocktail (Roche, Mannheim, Germany). After centrifugation at $15,000 \mathrm{rpm}$ for $30 \mathrm{~min}$ at $4{ }^{\circ} \mathrm{C}$, the supernatants were recovered. $12 \%$ SDS-PAGE was conducted with each of the native proteins (30 $\mu \mathrm{g}$ of each well) and $\mathrm{rSjGPx}$ protein $(50 \mathrm{ng})$ under reducing condition. Measurement of protein concentration was determined according to the procedure described by Bradford (1976) [33]. The gel was stained with Coomassie blue or transferred onto nitrocellulose membrane (Schleicher \& Schuell BioScience, Dassel, Germany). The membrane was blocked for $1 \mathrm{~h}$ in Tris-buffered saline containing $0.05 \%(\mathrm{v} / \mathrm{v})$ Tween-20 (TBS/T) and 5\% (w/v) skim milk, followed by overnight incubation with the anti-SjGPx antibody diluted to $1: 1,000$ in TBS/T containing $5 \%$ skim milk at $4{ }^{\circ} \mathrm{C}$. The membrane was subsequently incubated for additional $1 \mathrm{~h}$ at room temperature with horseradish peroxidase (HRP)-conjugated goat anti-mouse IgG antibody (1:2,000 dilutions; Cappel, West Chester, PA, USA). The blots were developed using an ECL system (Amersham Biosciences) under the manufacturer's protocol. 
2.7 Synthesis of dsRNA of SjGPx and treatment of Schistosomes in vitro culture

$S j G P x$-dsRNA was synthesized from cDNA encoding the full mature length of SjGPx using gene-targeted primers containing T7 promoter sequences

5'-TAATACGACTCACTATAGGGATGATTGATTGTTTCACTCGAG-3' and 5'-TAATACGACTCACTATAGGGCTATTTACTGGAAACGTCAAGC-3' spanning coding DNA positions of 55-630. One negative control dsRNA against Firefly luciferase (Luc-dsRNA) was also synthesized from plasmid pGL3-Basic (Promega, Madison, WI). RNAs were synthesized and purified using the Megascript RNAi kit (Ambion, Austin, TX) according to the manufacturer's instructions. dsRNAs were precipitated with $5 \mathrm{M}$ ammonium acetate and $95 \%$ ethanol, after which the RNA pellets were dissolved in water. Integrity of the dsRNAs was verified by non-denaturing $1 \%$ agarose gel electrophoresis, and concentration and purity determined with a spectrophotometer (ND-1000, NanoDrop Technologies, Wilmington, DE).

The Lipofectamine ${ }^{\mathrm{TM}} 2000$ transfection reagent (Invitrogen, USA) was used in the dsRNA selection experiment to improve transfection efficiency. $800 \mu \mathrm{l}$ of OPTI-MEM (Invitrogen, USA) containing $8 \mu \mathrm{l}$ of Lipofectamine ${ }^{\mathrm{TM}} 2000$ and dsRNA was added into 3.2 $\mathrm{ml}$ of antibiotic-free RPMI 1640 medium, in which 50 mated worms were cultured. Then the worms were cultured for $6 \mathrm{~h}$ at $37{ }^{\circ} \mathrm{C}$ before media were replaced with fresh RPMI 1640 medium containing fetal bovine serum and antibiotics [27].

To examine the optimal concentration of dsRNA in mediating SjGPx knockdown, $10 \mathrm{nM}$ and $50 \mathrm{nM}$ of the dsRNAs were added into the culture. After 5 days of cultivation, the parasites were collected for determination of $S j G P x$ transcript level by using semi-quantitative 
RT-PCR. The protein levels of SjGPx were determined by western blotting and a GPx activity assay, which was estimated by using a specific Glutathione Peroxidase Assay Kit (Northwest Life Science Specialties, LLC, Canada). The morphological characteristics of intrauterine eggs were observed by using a light microscope (Olympus, CKX41SF, Japan) from the 1st to 5th day.

\subsection{Induction of the S. japonicum GPx against oxidative stress}

Forty fresh intact adult worms were pre-incubated in $50 \mathrm{~mL}$ RPMI 1640 medium for $1 \mathrm{~h}$ at $37^{\circ} \mathrm{C}$. The worms were transferred into fresh media containing methyl viologen dichloride hydrate (paraquat, $10 / 50 \mathrm{mM}$ ) or $\mathrm{H}_{2} \mathrm{O}_{2}(0.2 / 1.0 \mathrm{mM})$ and incubated for $2 \mathrm{~h}$ at $37{ }^{\circ} \mathrm{C}[20,21]$. The worms were washed more than 10 times with cold physiological saline at $4{ }^{\circ} \mathrm{C}$. Soluble proteins were prepared from each of the experimental groups, as described above. Incubation with medium only was used as a control. The induction profile of SjGPx protein was examined via western blotting with specific antibody. An anti-SjTubulin antibody (currently available in our laboratory) was used as an internal standard, of which constant expression in response to the stimuli was verified by RT-PCR. Changes in the total GPx activity were estimated using the specific Glutathione Peroxidase Assay Kit (Northwest Life Science Specialties, LLC, Canada). The measurements were conducted with triplicate reactions and expressed as Means \pm S.D. of enzymatic unit/mL/mg protein.

\section{Results}

\subsection{Isolation of S. japonicum gene putatively encoding selenium-dependent PHGPX}


The full-length SjGPx transcript was comprised of 898 bp, and contained 576 bp open reading frame, from an ATG at nucleotide position 55-57 to a TAG at position 628-630. In addition to the conventional stop codon, $S j G P x$ gene contained a second in-frame stop codon within its ORF at nucleotide position 241-243, which might encode the 21st aa, Sec [34]. In association with the presence of the opal codon, a SECIS motif was detected at nucleotide positions 697-792 within the 3'-untranslated region (UTR) (Fig. 1). The SECIS conserved several structural motifs characteristic to those of selenium-dependent homologues, including 2 helices separated by an internal loop, a SECIS core structure, a quartet located at the base of the second helix and an apical loop; all of which have been well described in a series of GPx genes [35]. The full-length cDNA sequence of $S j G P x$ was registered in GenBank under Accession no. KJ941009.

\subsection{Determination of primary structure of SjGPx}

The decoded polypeptide of SjGPx was found to be composed of 191 amino acids with predicted $\mathrm{MW} / \mathrm{pI}$ of $21.74 \mathrm{kDa} / 8.22$, and shared a significant degree of sequence identity with other PHGPx proteins (34\% 77\%, Supplementary Fig. 1). The 20 aa residues encompassing the N-terminus of SjGPx was found to be hydrophobic and predicted as target signals for the translocation of the proteins (underlined sequence in Supplementary Fig. 1). SjGPx contained three conserved functional domains found in the GPx family and the aa residues constituting the catalytic triad (Sec, Gln, and Trp) were positioned separately within each of the domains (filled arrowhead in domain A, B and C, Supplementary Fig. 1). Among the 3 aa residues involved in the formation of a salt bridge and hydrogen bond to the glutathione molecule [11], 
only the Gln and downstream Arg residues were shown to be highly conserved among members of PHGPx, including SjGPx (open arrowheads in Supplementary Fig. 1). The subunit interaction domains, which is required for the formation of an active tetrameric structure in mammalian GPx isozymes other than PHGPxs [12], was not detected in the aa sequence of SjGPx (data not shown). The aa sequence of SjGPx was registered in GenBank under Accession no. AJD79087.

\subsection{Phylogenetic relationships of SjGPx with their homologues}

The aa sequence of SjGPx was used in homology searches with BLAST algorithms from the GenBank database, and several hundred PHGPx-like proteins were retrieved. These sequences revealed various degrees of sequence identity to SjGPx ranging 35\%-53\% (E-values<1e-20). Information regarding the other GPx families was obtained by a series of subsequent BLAST searches using the aa sequence of human GPx3 (AAP50261). A total of 48 entries were finally selected, by considering both redundancy and taxonomical distributions and used in a phylogenetic analysis. As shown in Fig. 2, six classes of the GPx family were well separated in the phylogenetic tree constructed by the neighbor joining (NJ) method. A similar clustering pattern was also observed in a tree based on the maximum parsimony algorithm, of which bootstrap values were incorporated into the NJ tree. The members of PHGPx had a highly diverse donor source and the GPxs identified from free-living nematodes, insects and plants, as well as trematodes, were categorised into this group. The tree revealed that each of the trematode and insect PHGPxs had diverged separately into two distinct subclades. The SjGPx was clustered into the trematode Subclade 
II together with S. mansoni GPx2 (AAU34080). The Sec codon and the associated SECIS motif were recognized exclusively within the mRNA sequences coding for the trematode and vertebrate GPx genes (indicated by $\dagger$ and $\ddagger$, respectively, in Fig. 2). Among the arthropod proteins examined, only an arachnidal tick PHGPx (Boophilus microplus, ABA25916) was found to be Sec-dependent.

\subsection{Expression patterns of SjGPx gene and its expression protein}

Expression levels of the SjGPx transcript were examined by semi-quantitative RT-PCR using RNAs isolated from S. japonicum egg, cercariae, male and female adult. As shown in Fig. 3A, the amplification of the target gene clearly revealed that this Schistosoma gene was highly transcribed in the egg and mature female worm. The gene of cercariae and adult male worm showed very low transcription levels. The S. japonicum alpha-tubulin gene (Sj-Tubulin), used as an internal control, exhibited a constant expression level during the stages (Fig. 3A). A mature domain of SjGPX was amplified, and cloned into the pET-28a prokaryotic expression vector. The in vitro expressed rSjGPx protein was found as soluble form, and then purified with Ni-NTA agarose chromatography (Supplementary Fig. 2). To observe the native SjGPx protein, we conducted western blotting, employing the egg, cercariae and adult worm extracts, using anti-SjGPx specific mouse sera (Fig. 3B). The cercariae proteins were not found to react with antibody to SjGPx. However, the blot containing the adult and egg whole proteins, which had been extracted in the presence of $2 \%$ SDS, exhibited strong positive reactions. The results were well matched to the mRNA expression levels. As shown in Fig. 3B, A strong band (migrated approximately $21 \mathrm{kDa}$ ), which might be well matched with the size 
of SjGPx protein, was recognized by SjGPx antiserum. As a control, SjGPx was reactive with the rSjGPx protein.

\subsection{RNAi-mediated knockdown of SjGPx and morphological observation of intrauterine eggs}

Effects of RNAi-mediated knockdown on SjGPx were examined by using semi-quantitative RT-PCR, western blotting and GPx activity assays. As shown in Fig. 4, the effect of dsRNA was dose-dependent. $50 \mathrm{nM} S j G P x$ dsRNA significantly reduced the transcript level of the target gene (Fig. 4A). The reduced level of SjGPx protein caused by dsRNA in schistosomes paralleled reduction in mRNA level (Fig. 4B). The whole GPx enzyme activities were reduced approximately $27.68 \%$ and $53.86 \%$ after adding $10 \mathrm{nM}$ and $50 \mathrm{nM}$ dsRNA, respectively, as shown in Fig. 4C. From the 1st to 5th day after SjGPX gene knockdown, the morphology of intrauterine eggs was continuously observed under a light microscope. On the 1 st to 2 nd day, the intrauterine eggs maintained a normal shape. On the 3rd day post-treatment, intrauterine eggs resembling spindles began to appear in the SjGPX gene knockdown group (Fig. 4D), and on the 5th day, the eggs from intrauterine of females and eggs from culture medium were more severely deformed. Irregular eggs, including spindle-like eggs and eggs with invaginated eggshells adhering to each other were observed in the female uteri (Fig. 4D). The majority of eggs in uteri of females and in culture medium from the untreated group (Blank) and negative control group (Luc-dsRNA) were morphologically normal. 
worms

In order to assess the induction profiles of SjGPx during oxidative stress, we treated viable $S$. japonicum adult worms with paraquat or $\mathrm{H}_{2} \mathrm{O}_{2}$ for $2 \mathrm{~h}$ at $37{ }^{\circ} \mathrm{C}$. As shown in Fig. 5A, western blotting indicated that these stimuli substantially augmented the expression levels of the SjGPx protein. A quantitative calorimetric assay also demonstrated dose-dependent increase of the enzyme activity. In the control group of un-treated with chemicals, the relative enzymatic activity of SjGPx proteins in whole adult worm extracts was $2.76 \pm 0.12 \mathrm{U} / \mathrm{ml} / \mathrm{mg}$. Enzyme activities were induced approximately 1.6 - to 2.1 -fold after adding $10 \mathrm{mM} / 50 \mathrm{mM}$ paraquat or $0.2 \mathrm{mM} / 1.0 \mathrm{mM} \mathrm{H} \mathrm{O}_{2}$ (Fig. 5B).

\section{Discussion}

PHGPx is a major antioxidant enzyme, which plays crucial roles in the protection of cells against oxidative stress by catalysing reduction of lipid hydroperoxides and in the repairing of disrupted biomembranes by directly reducing hydroperoxidized phospholipids integrated into membranes [13]. In the present study, we isolated and characterised a $P H G P x$ gene from a blood fluke $S$. japonicum. Many PHGPx proteins have been known to contain an opal UGA codon, encodes $\mathrm{Sec}$ at their catalytic site. The alternative decoding of UGA generally depends on a cis-factor, called SECIS, which is located in the 3'-UTR of these selenoprotein genes [34]. Our results demonstrated that SjGPx gene is a selenium-dependent PHGPx, which contain Sec codon and SECIS motif within its mRNA sequence (Fig. 1). A biased distribution of Sec-dependent PHGPxs was detected along with the taxonomical positions of their donor species (Supplementary Fig. 1 and Fig. 2). The Sec codon and 
concurrent SECIS motif were exclusively identified within the mRNA sequences of trematode and vertebrate GPx genes. Among the trematode GPx enzymes obtainable, only a $P$. westermani GPx (ABE68811) was found to lose Sec-related sequence factors and was therefore determined to be a selenium-independent GPx (Supplementary Fig. 1).

PHGPx proteins have been isolated from most eukaryotes, including vertrbrates, insects, nematodes, trematodes and plants, whereas the other GPx isoenzymes show a more restricted taxonomical distribution. In addition to vertebrates, a number of filarial nematodes are known to express GPx3-like enzymes [16]. Among the reported trematodes, two GPx proteins in $S$. mansoni, four GPx proteins in C. sinensis, and two GPx proteins in P. westermani, showing strong phylogenetic affinities toward the vertebrate PHGPx, have been described. These PHGPx proteins of trematodes were diverged into two distinct sub-clades [20]. In this study, the deduced aa sequence of SjGPx also revealed the primary structure characteristic to the PHGPx (GPx4) family, including amino acid conservation, absence of the subunit interaction domain and well-preserved catalytic and glutathione-binding domains (data not shown). In a phylogenetic analysis, SjGPx protein also showed tight relationships with the other PHGPX-related members (Fig. 2).

Helminth parasites may be continuously exposed to multiple oxidative stresses, including aerobic respiration, immune defense, and other oxidative pathways $[15,36] . \mathrm{H}_{2} \mathrm{O}_{2}$ and its subproducts are probably the most important ROS in biological systems, and directly affect different groups of biomolecules found in cells, such as lipids, proteins, and nucleic acids [37]. The main changes that occur during oxidative stress are the formation of protein carbonyls and the breaking of disulfide bonds, which renders impossible the maintenance of 
the native protein conformation [38]. Therefore, during the parasites life-span in host tissues, removal of ROS and protection of macromolecules from the oxidative attacks would be inevitable. The antioxidant enzymes might also constitute a major defensive system in these causative parasites [39]. The adults of S. japonicum settle in the mesenteric veins, and their eggs lodge in the fine venules of intestine wall or are carried retrograde by the portal venous system to the liver. The parasite might be continuously exposed to oxidative stresses. In order to overcome these stressful conditions and to maintain its life-span, the parasite is equipped with the antioxidant enzymes. Furthermore, phenol oxidase, which is secreted from the vitellocytes, acts on eggshell precursors, thus converting tyrosine into quinone in the presence of oxygen. During this tanning process, incomplete reduction of the oxygen may result in a local increase of harmful ROS [40,41]. Therefore, effective antioxidant enzymes, such as GPx, might be required for the protection of macromolecules and/or the fertilised ovum. Among the reported trematode GPx enzymes, S. mansoni GPx proteins were previously shown to be localized on vitelline follicles and eggs [22]. The PHGPX-like proteins from $C$. sinensis and $P$. westermani parasites, were also found localised in vitellocytes within vitelline follicles and premature eggs [20,21]. In the present study, our RT-PCR and western blotting results showed that the S. japonicum GPx expression was found to be confined exclusively to the adult female worms and eggs (Fig. 3). These results and above analysis indicated that trematode GPx proteins might play key roles during the parasite egg production/eggshell formation.

Increasing evidence indicates that loss-of-function genetic manipulation through RNAi may be generally applicable for investigation of schistosome genes [30,42-43]. In schistosomes this can result in long-term knockdown and silencing of the target gene [30]. To 
investigate the involvement of the GPx gene of schistosome egg formation, we performed a RNAi experiment, delivering SjGPx dsRNA into schistosomes by Lipofectamine transfection assay. The results of RNAi-mediated knockdown of SjGPX in transcript showed that the GPx enzymatic activities were specifically suppressed, and the undeveloped intrauterine eggs of females were more severely deformed. Irregular eggs were observed in the female uteri (Fig. 4D). Therefore, effective antioxidant enzymes, such as GPx, may be required for the protection of macromolecules and/or the fertilized ovum during the trematode egg production/maturation. Gene knockdown of SjGPX mRNA might be represent a novel and potentially effective strategy for combating schistosomiasis.

We demonstrated that oxidative stress can induce SjGPx activity approximately 1.6- to 2.1-fold (Fig. 5). However, the enzyme activity declined at the high concentration of $\mathrm{H}_{2} \mathrm{O}_{2}(2$ $\mathrm{mM})$, which might be attributed to a lethal effect of this chemical on the worms $(60-70 \%$ worms survived after the treatment, data not shown). GPxs of $C$. sinensis and S. mansoni also showed a similar effect in response to $\mathrm{H}_{2} \mathrm{O}_{2}$, although the induction levels were not significantly increased compared with those of the prominent PRxs [44]. These results may suggest that the trematode GPxs are engaged in antioxidant functions specifically in relation to egg production rather than the global regulation of redox-status, which might result from the regulation of phase I antioxidant genes induced by exogenous oxidative stress via Nrf2-mediated signalling pathway [45]. Similar to C. sinensis and S. mansoni GPxs, we did not observe any detectable enzymatic activity with the mutated $\mathrm{rSjGPx}$ protein expressed in bacteria cells (data not shown). Generation of Sec-containing active enzymes using eukaryotic systems will be warranted to define the detailed biochemical kinetics of trematode GPxs. 


\section{Acknowledgements}

This work was supported by a National Science Foundation of China (No. 30972568), a

research grant from Health Department of Hubei Province, P. R. China (No. XF2010-17) and a research found from Wuhan University (No. 2042015kf1036).

\section{References}

[1] Lei Z, Zheng H, Zhang L, Zhu R, Guo J, Li S, Wang L, Chen Z, Zhou X. Schistosomiasis situation in People's Republic of China in 2010. Chin J Schisto Control 2011;23:599-604 (in Chinese).

[2] Wang LD, Utzinger J, Zhou XN. Schistosomiasis control: experiences and lessons from China. Lancet 2008;372:1793-95.

[3] Wyler DJ. Why does liver fibrosis occur in schistosomiasis? Parastiol. Today 1992;8:277-9.

[4] Kazura JW, Fanning MM, Blumer JL, Mahmoud AA. Role of cell-generated hydrogen peroxide in granulocyte-mediated killing of schistosomula of Schistosoma mansoni in vitro. J Clin Invest 1981;67:93-102.

[5] Forman HJ, Torres M, Fukuto J. Redox signaling. Mol Cell Biochem 2002;234-235:49-62.

[6] Dröge W. Free radicals in the physiological control of cell function. Physiol Rev 2002;82:47-95.

[7] Sies H. Strategies of antioxidant defense. Eur J Biochem 1993;215:213-9.

[8] Mkoji GM, Smith JM, Prichard RK. Antioxidant systems in Schistosoma mansoni: correlation between susceptibility to oxidant killing and the levels of scavengers of hydrogen peroxide and oxygen free radicals. Int J Parasitol 1988;18:661-6.

[9] LoVerde PT, Carvalho-Queiroz C, Cook R. Vaccination with Antioxidant Enzymes Confers Protective Immunity against Challenge Infection with Schistosoma mansoni. Mem Inst Oswaldo Cruz 2004;99:37-43.

[10] Arthur JR. The glutathione peroxidases. Cell Mol Life Sci 2000;57:1825-35.

[11] Epp O, Ladenstein R, Wendel A. The refined structure of the selenoenzyme glutathione peroxidase at 0.2-nm resolution. Eur J Biochem 1983;133:51-69.

[12] Brigelius-Flohé R, Aumann KD, Blöcker H, Gross G, Kiess M, Klöppel KD, Maiorino M, Roveri A, Schuckelt R, Ursini F, Wingender E, Flohén L. Phospholipid-hydroperoxide glutathione peroxidase. Genomic DNA, cDNA, and deduced amino acid sequence. J Biol Chem 1994;269:7342-8.

[13] Ursini F, Bindoli A. The role of selenium peroxidases in the protection against oxidative damage of membranes. Chem Phys Lipids 1987;44:255-76.

[14] Imai H, Nakagawa Y. Biological significance of phospholipids hydroperoxide glutathione peroxidase (PHGPx, GPx4) in mammalian cells. Free Radic Biol Med 2003;34:145-69. 
[15] Zelck UE, von Janowsky B. Antioxidant enzymes in intramolluscan Schistosoma mansoni and ROS-induced changes in expression. Parasitology 2004;128:493-501.

[16] Henkle-Dührsen K, Kampkötter A. Antioxidant enzyme families in parasitic nematodes. Mol. Biochem. Parasitol 2001;114:129-42.

[17] Williams DL, Pierce RJ, Cookson E, Capron A. Molecular cloning and sequencing of glutathione peroxidase from Schistosoma mansoni. Mol Biochem Parasitol 1992;52:127-30.

[18] Mei H, LoVerde PT. Schistosoma mansoni: cloning the gene encoding glutathione peroxidase. Exp Parasitol 1995;80:319-22.

[19] Mei H, Thakur A, Schwarts J, LoVerde PT. Expression and characterization of glutathione peroxidase activity in the human blood fluke Schistosoma mansoni. Infect Immun 1996;64:4299-306.

[20] Cai GB, Bae YA, Kim SH, Sohn WM, Lee YS, Jiang MS, Kim TS, Kong Y. Vitellocye-specific expression of phospholipid hydroperoxide glutathione peroxidases in Clonorchis sinensis. Int J Parasitol 2008;38:1613-23.

[21] Kim SH, Cai GB, Bae YA, Lee EG, Lee YS, Kong Y. Two novel phospholipid hydroperoxide glutathione peroxidase genes of Paragonimus westermani induced by oxidative stress. Parasitology 2009;136:553-65.

[22] Roche C, Liu JL, LePresle T, Capron A, Pierce RJ. Tissue localization and stage-specific expression of the phospholipid hydroperoxide glutathione peroxidase of Schistosoma mansoni. Mol Biochem Parasitol 1996;75:187-95.

[23] Bosher JM, Labouesse M. RNA interference: genetic wand and genetic watchdog. Nat Cell Biol 2000;2:E31-6.

[24] Mourão MM, Dinguirard N, Franco GR, Yoshino TP. Phenotypic screen of earlydeveloping larvae of the blood Fluke, Schistosoma mansoni, using RNA interference. PLoS Negl Trop Dis 2009;3:e502.

[25] Cheng G, Fu Z, Lin J, Shi Y, Zhou Y, Jin Y, Cai Y. In vitro and in vivo evaluation of small interference RNA-mediated gynaecophoral canal protein silencing in Schistosoma japonicum. J Gene Med 2009;11:412-21.

[26] Tchoubrieva EB, Ong PC, Pike RN, Brindley PJ, Kalinna BH. Vector-based RNA interference of cathepsin B1 in Schistosoma mansoni. Cell Mol Life Sci 2010;67:3739-48.

[27] He Y, Cai G, Ni Y, Li Y, Zong H, He L. siRNA-mediated knockdown of two tyrosinase genes from Schistosoma japonicum cultured in vitro. Exp Parasitol 2012;132:394-402.

[28] Zamore PD, Tuschl T, Sharp PA, Bartel DP. RNAi: double-stranded RNA directs the ATP-dependent cleavage of mRNA at 21 to 23 nucleotide intervals. Cell 2000;101:25-33.

[29] Milhavet O, Gary DS, Mattson MP. RNA interference in biology and medicine. Pharmacol Rev 2003;255:629-48.

[30] Morales ME, Rinaldi G, Gobert GN, Kines KJ, Tort JF, Brindley PJ. RNA interference of Schistosoma mansoni cathepsin D, the apical enzyme of the hemoglobin proteolysis cascade. Mol Biochem Parasitol 2008;157:160-8.

[31] He B, Cai G, Ni Y, Li Y, Zong H, He L. Characterization and expression of a novel cystatin gene from Schistosoma japonicum. Mol Cell Probes 2011;25:186-93.

[32] Liu LY, Yu XB, Luo SH, Fang JM. Quick isolation and purification of Schistosoma 
japonicum eggs and their total RNA. Chin J Zoonoses 1997;13:17-9 (in Chinese).

[33] Bradford MM. Rapid and sensitive method for the quantitation of microgram quantities of protein utilizing the principle of protein-dye binding. Anal Biochem 1976;72:248-54.

[34] Stadtman TC. Selenocysteine. Annu Rev Biochem 1996;65:83-100.

[35] Kryukov GV, Castellano S, Novoselov SV, Lobanov AV, Zehtab O, Guigó R, Gladyshev VN. Characterization of mammalian selenoproteomes. Science 2003;300:1439-43.

[36] Selkirk ME, Smith VP, Thomas GR, Gounaris K. Resistance of filarial nematode parasites to oxidative stress. Int J Parasitol 1998;28:1315-32.

[37] Corcoran A, Cotter TG. Redox regulation of protein kinases. FEBS J 2013;280:1944-65.

[38] Kastle M, Grune T. Protein oxidative modification in the aging organism and the role of the ubiquitin proteasomal system. Curr Pharm Des 2011;17:4007-22.

[39] Callahan HL, Crouch RK, James ER. Helminth anti-oxidant enzymes: a protective mechanism against host oxidants? Parasitol Today 1988;4:218-25.

[40] Cai G, Bae Y, Zhang Y, He Y, Jiang M, He L. Expression and characterization of two tyrosinases from the trematode Schistosoma japonicum. Parasitol Res 2009;104:601-9.

[41] Bae YA, Kim SH, Ahn CS, Kim JG, Kong Y. Molecular and biochemical characterization of Paragonimus westermani tyrosinase. Parasitology 2015;142:807-15.

[42] Krautz-Peterson G, Radwanska M, Ndegwa D, Shoemaker CB, Skelly PJ. Optimizing gene suppression in schistosomes using RNA interference. Mol Biochem Parasitol 2007;2:194-202.

[43] Tavernarakis N,Wang SL, Dorovkov M, Ryazanov A, Driscoll M. Heritable and inducible genetic interference by double-stranded RNA encoded by transgenes. Nat Genet 2000;2:180-3.

[44] Sayed AA, Cook SK, Williams DL. Redox balance mechanisms in Schistosoma mansoni rely on peroxiredoxins and albumin and implicate peroxiredoxins as novel drug targets. $\mathbf{J}$ Biol Chem 2006;281:17001-10.

[45] Rushmore TH, Kong AN. Pharmacogenomics, regulation and signaling pathway of phase I and II drug metabolizing enzymes. Curr Drug Metab 2002;3:481-90. 


\section{Legends for Figures}

Fig. 1 Analysis of selenocysteine insertion sequence (SECIS) elements in phospholipid hydroperoxide glutathione peroxidase (PHGPx) gene of Schistosoma japonicum. (A) The secondary structure SECIS elements found in the 3'-untranslated region (UTR) of SjGPX mRNA. The structure was determined by using the SECISearch program (ver2.19). (B) The primary structures of the SECIS motif. Nucleotide sequences constituting the 3'-UTR SECIS motifs were retrieved from the PHGPx genes of Clonorchis sinensis, Paragonimus westermani, Schistosoma mansoni, Homo sapiens, and Mus musculus, and aligned in accordance with the predicted secondary elements. Nucleotides conserved within the invariant quartet and apical loop are highlighted in boldface letters.

Fig. 2 Phylogenetic relationships of SjGPx among various members of glutathione peroxidase (GPx) families. A phylogenetic analysis was conducted based on a multiple alignment of amino acid sequences retrieved from the GenBank database. The tree was constructed by a maximum parsimony algorithm using PHYLIP after excluding positions with gaps and was unrooted. Numbers at branching nodes indicate their percentages of appearance in 100 bootstrap replicates. The branches separating each of the GPx families are presented with thick bars. The presences of selenocysteine (Sec) codon $(\dagger)$ and Sec insertion sequence $(\dagger)$ within each of the corresponding mRNA sequences are indicated at the end of each sequence names. The identity of each analysed sequence is distinguished by a protein GenBank accession number, followed by the species name from which it has been isolated.

Fig. 3 Identification of the native SjGPx in Schistosoma japonicum. (A) Transcription profile of SjGPx gene in S. japonicum parasite. The transcription level of SjGPx was determined with the gene-specific primers via a semi-quantitative RT-PCR. Total RNAs were extracted from egg, cercariae, male and female adult stages of the parasite, and used as templates to examine $S j G P x$ transcripts. The amplified products were separated on $2 \%$ agarose gels and stained with ethidium bromide. The S. japonicum alpha-tubulin (Sj-Tubulin, AY815746) was also amplified using the specific primers as an internal control. The absence of any contaminated genomic DNA was confirmed by preparing reactions without reverse transcriptase during the first reverse transcription step (RT-). The position of 100-bp DNA size standard (M) was shown on the left. (B) Western blotting of the native SjGPx protein. A total of $30 \mu \mathrm{g}$ of proteins extracted from adults, cercariae and egg were separated on a $12 \%$ SDS-PAGE gel under reducing conditions and transferred onto nitrocellulose membranes. The membranes were immuno-reacted against the recombinant SjGPx -specific mouse antisera. The rSjGPx (50 ng) was employed as positive control. Molecular weight marker $(\mathrm{Mr})$ in kDa was shown on the left.

Fig. 4 Inhibitory effects of dsRNA-mediated gene knockdown of SjGPx. 0 nM, $10 \mathrm{nM}$ and 50 $\mathrm{nM}$ of the dsRNAs were added into the schistosomes culture. After 5 days of RNAi, mated worms were collected for determination of (A) SjGPx transcript level by using semi-quantitative RT-PCR with the gene-specific primers; (B) SjGPx protein level by using western blotting with the SjGPx-specific mouse antisera; and (C) the GPx activity by using a 
Glutathione Peroxidase Assay Kit, the assays were conducted in triplicate employing the worm extracts $\left(10 \mu \mathrm{g}\right.$ of each) and expressed as mean $\pm \mathrm{SD} .{ }^{*} \mathrm{P}<0.05$ by Student's t test. (D) RNAi effects of $50 \mathrm{nM} \mathrm{SjGPx}$ dsRNA on intrauterine eggs formation in Schistosoma japonicum female adult worms cultured in vitro for 5days. Intrauterine eggs within female adult worms and laid eggs from the culture medium in the untreated group (Blank), negative control group (Luc-dsRNA) and SjGPx group (SjGPx-dsRNA) were observed using a light microscope under the common light.

Fig. 5 Induction of SjGPx upon exogenous stimulation with oxidative chemicals. (A) Intact viable adult worms were incubated for $1 \mathrm{~h}$ at $37{ }^{\circ} \mathrm{C}$ in RPMI 1640 media, after which the worms were further incubated for $2 \mathrm{~h}$ in fresh media supplemented with the respective chemicals. The level of SjGPx expression was examined by western blotting. An antibody specific to the Schistosoma japonicum Tubulin ( $\mathrm{Sj}$-Tubulin) was used as an internal standard. (B) GPx activity in the parasite extracts under normal and oxidative stress conditions. The specific activity was spectrometrically measured using a specific GPx assay kit, as described in the Section 2. The specific enzymatic activity $(\mathrm{U} / \mathrm{ml} / \mathrm{mg})$ was calculated using the equation, $[\mathrm{GPx}]=\left\{2\left(\mathrm{mRate}_{\mathrm{s}}-\mathrm{mRate}_{\mathrm{b}}\right) \cdot \mathrm{V}_{\mathrm{Rxm}} / 2.74 \cdot \mathrm{V}_{\mathrm{s}}\right\} \cdot \mathrm{df}$, the assays were conducted in triplicate employing the worm extracts $\left(10 \mu \mathrm{g}\right.$ of each) and expressed as mean $\pm \mathrm{SD}$. ${ }^{*} \mathrm{P}<0.05$ by Student's t test. 
A

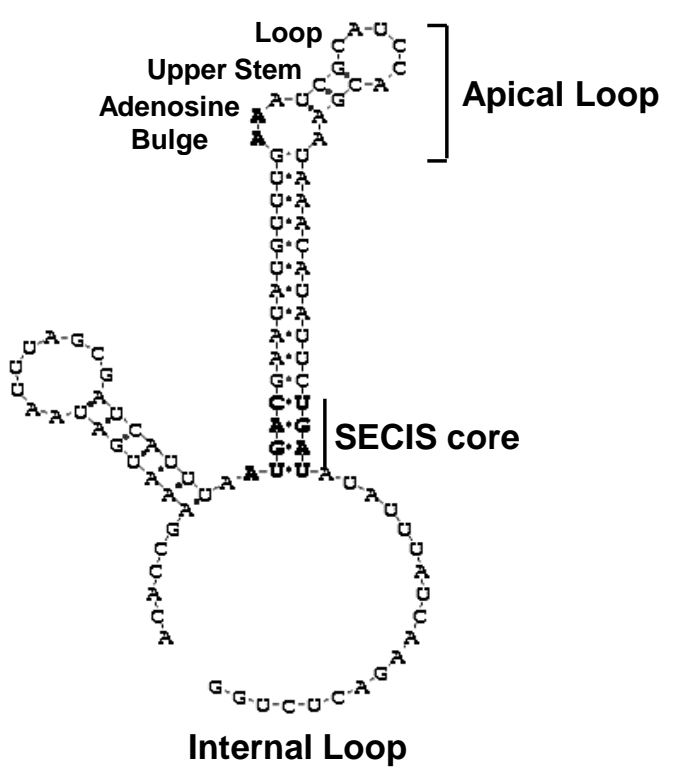

\begin{tabular}{|c|c|c|c|c|c|c|c|c|c|c|}
\hline Organism & $\begin{array}{l}\text { GenBank } \\
\text { Number }\end{array}$ & Helix I & $\begin{array}{l}\text { Internal } \\
\text { Loop 5' }\end{array}$ & $\begin{array}{c}\text { Quarter } \\
\text { (SECIS core) }\end{array}$ & Helix II & Apical Loop & Helix II & $\begin{array}{c}\text { Quarter } \\
\text { (SECIS core) }\end{array}$ & $\begin{array}{l}\text { Internal } \\
\text { loop 3' }\end{array}$ & Helix I \\
\hline S. japonicum & KJ941009 & AUCA & UUUA & A UGAC & GAA//UUG & AA AUC//GAA & U̇AA/UUC & UGAU & $\mathrm{AUA} / / \mathrm{UGG}$ & ÜGAU \\
\hline C. sinens & EF056481 & AGCC & GUC//CCU & A UGAA & GGC//CCU & CA AAC//GUU & $\mathrm{ACC}$ & UGAU & GUC & GGCU \\
\hline P. westermani & DQ454160 & AUCGC & ACG//UUA & A UGAA & AAA//UCG & AA GUCUACCACA & $\mathrm{CGG} / / \mathrm{UUC}$ & UGAU & u & GCGAU \\
\hline S. mansoni & M86510 & ACAAA & UUC//UAU & A UGAC & GAU//CUC & AA AUGUUCAUU & GUC//GGU & UGAU & GAA//AGU & UUUGU \\
\hline H. sapiens & X71973 & GCC & ACUC & A UGAC & GGC//UGC & AA ACCU & GCU//GGC & AGAC & $\mathrm{CCG} / / \mathrm{CAG}$ & GGC \\
\hline M. musculus & D87896 & GCC & ACUC & A UGAA & GGU//UGA & AA ACC//GUG & GGG//UCC & UGAG & GACCU & GGC \\
\hline
\end{tabular}

\section{Zhang et al-Fig. 1}




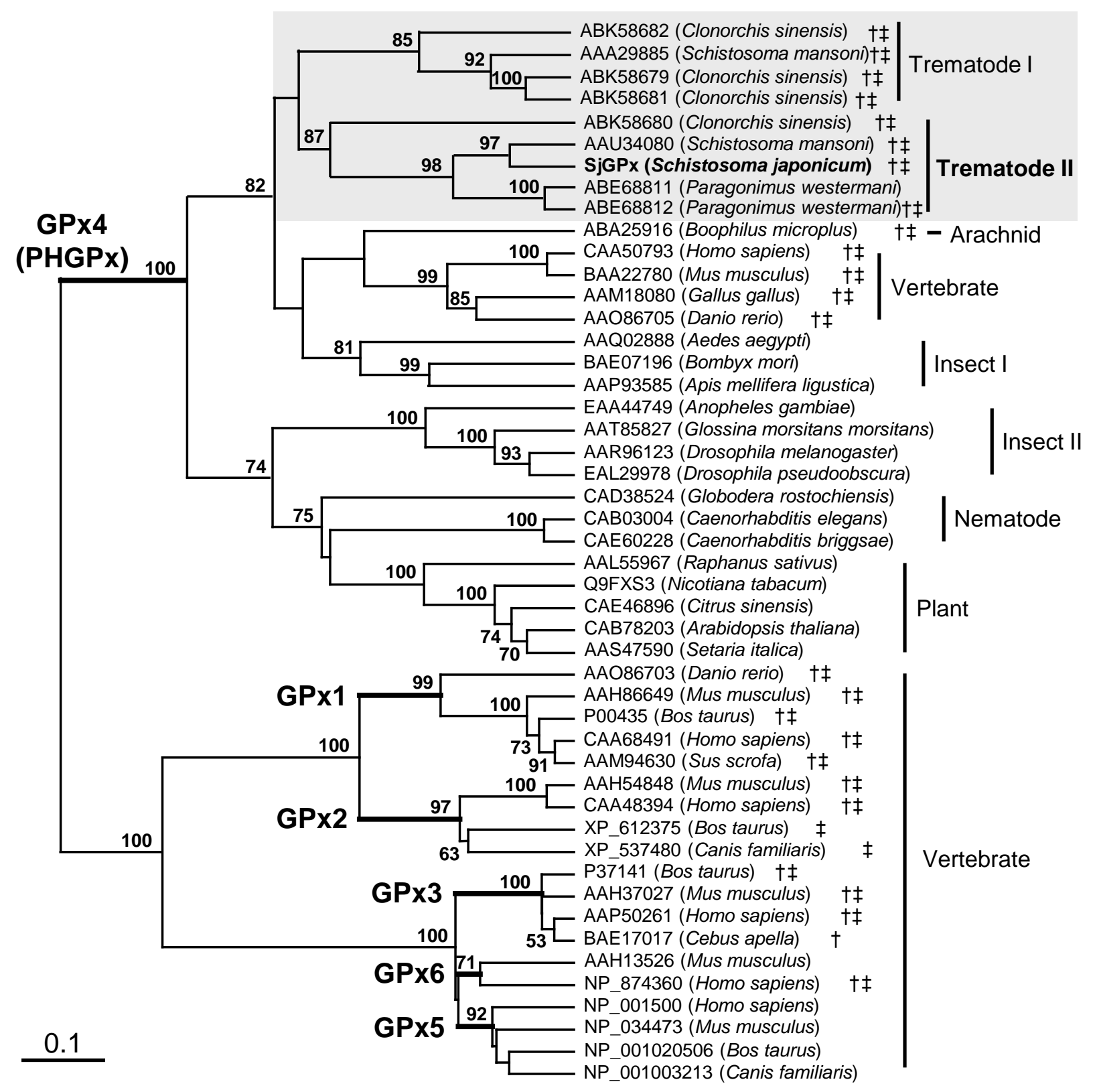

Zhang et al-Fig. 2 
A
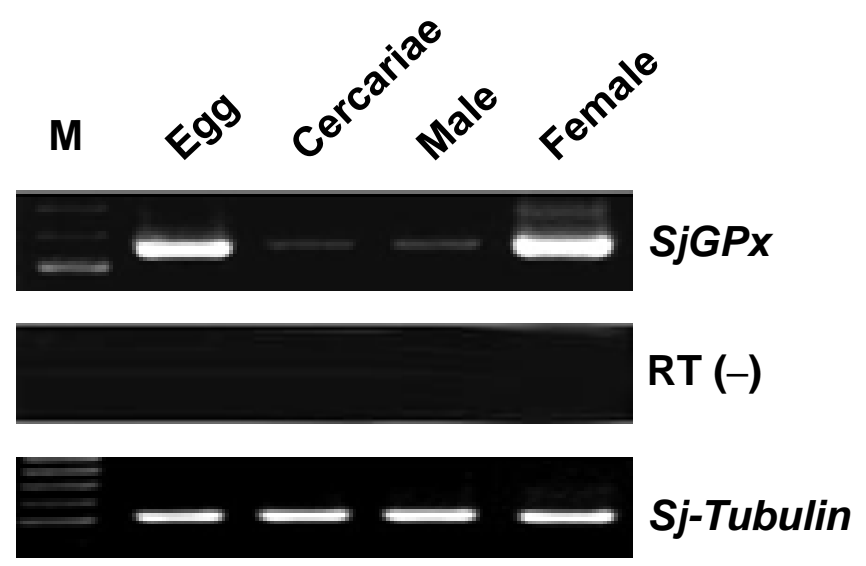

RT-PCR
B

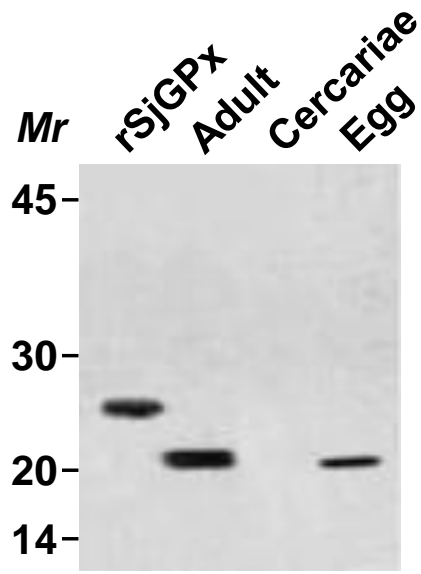

Western blotting 
A

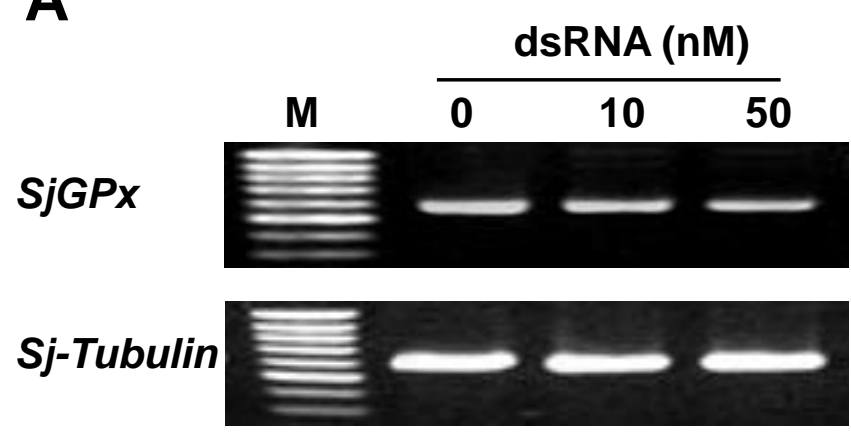

RT-PCR

D
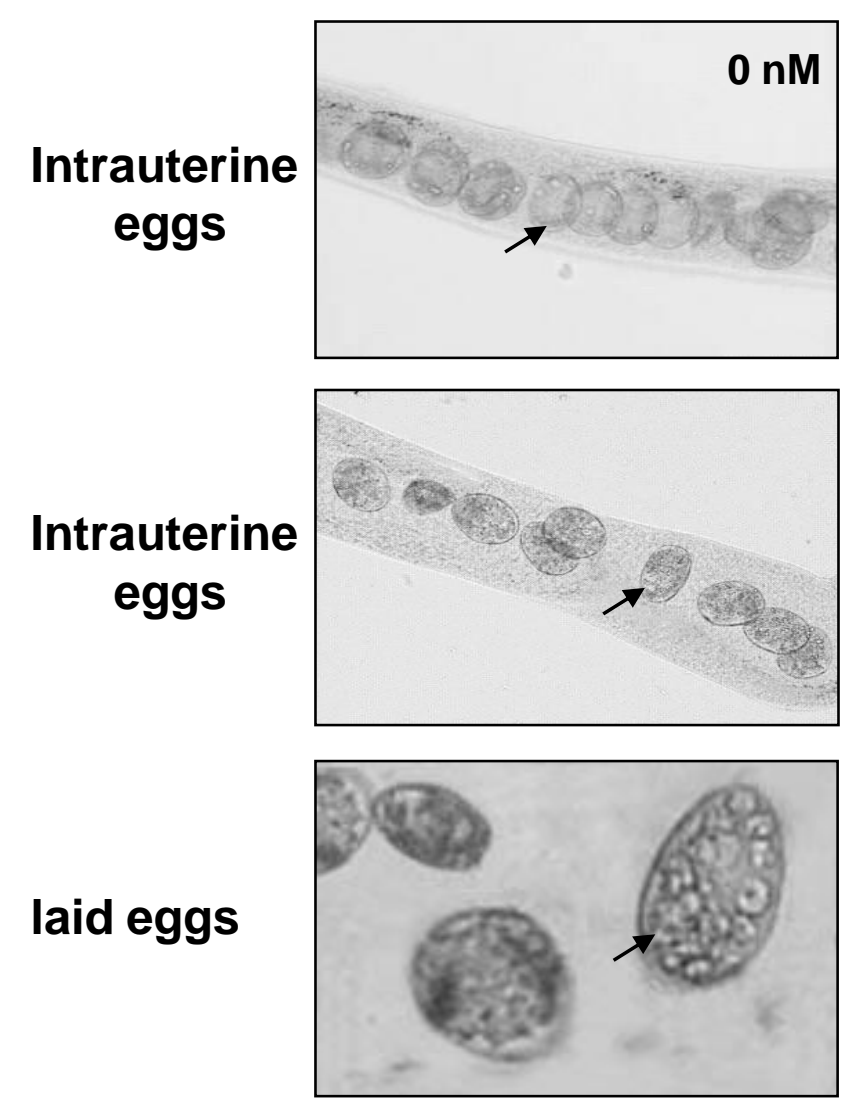

Zhang et al-Fig. 4
B

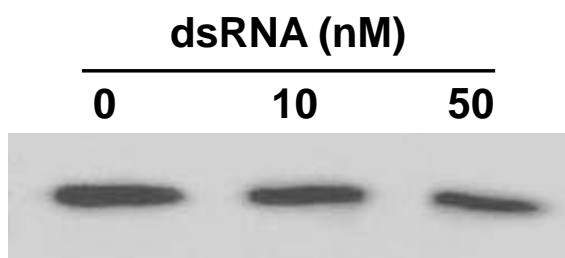

Western blotting
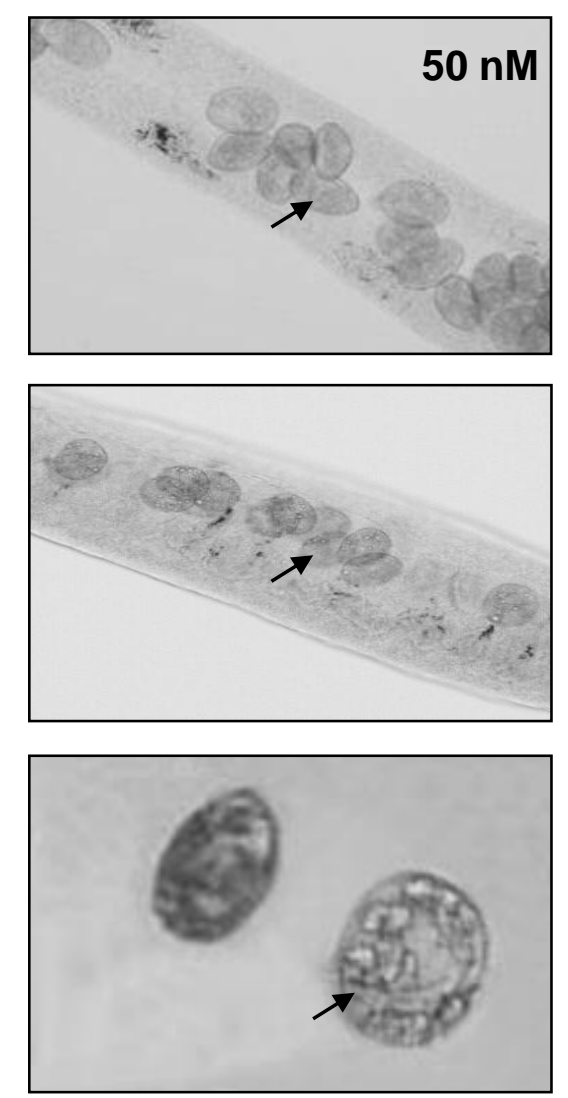

Luc-dsRNA
C $\quad \operatorname{dsRNA}(\mathrm{nM})$
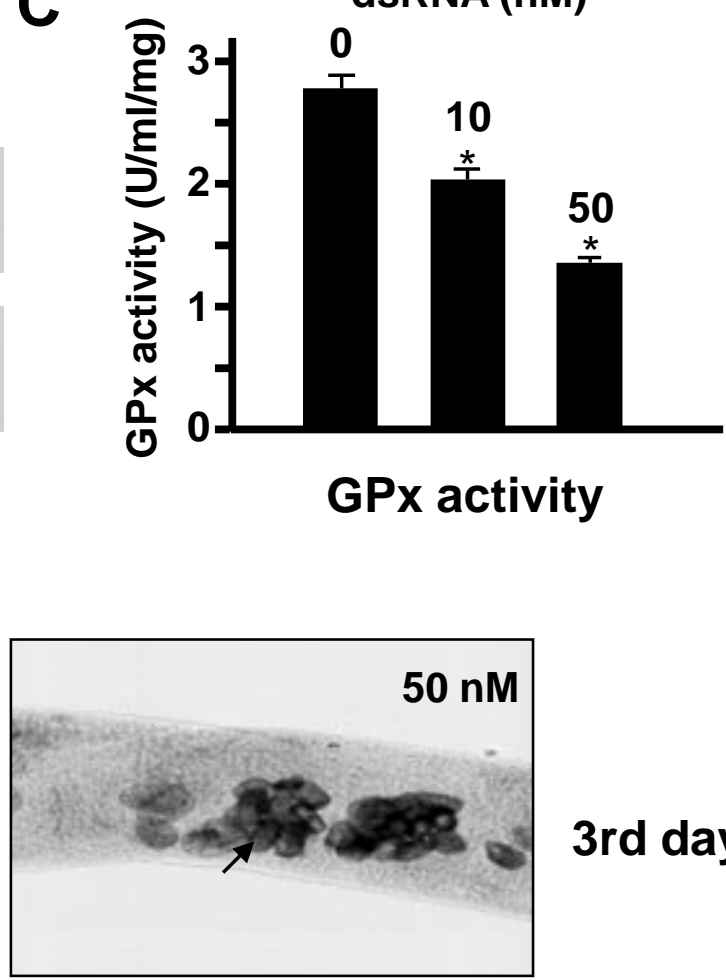

3rd day

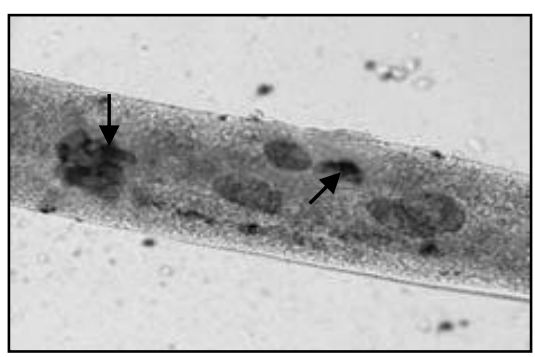

5th day

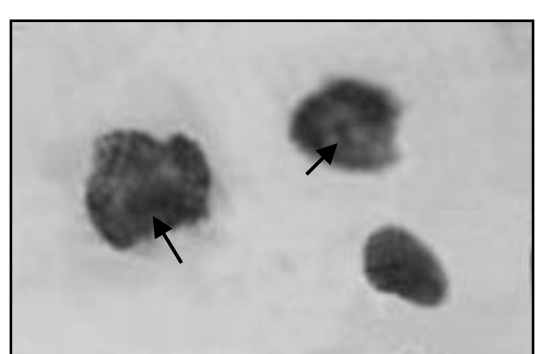

SjGPx-dsRNA 
A

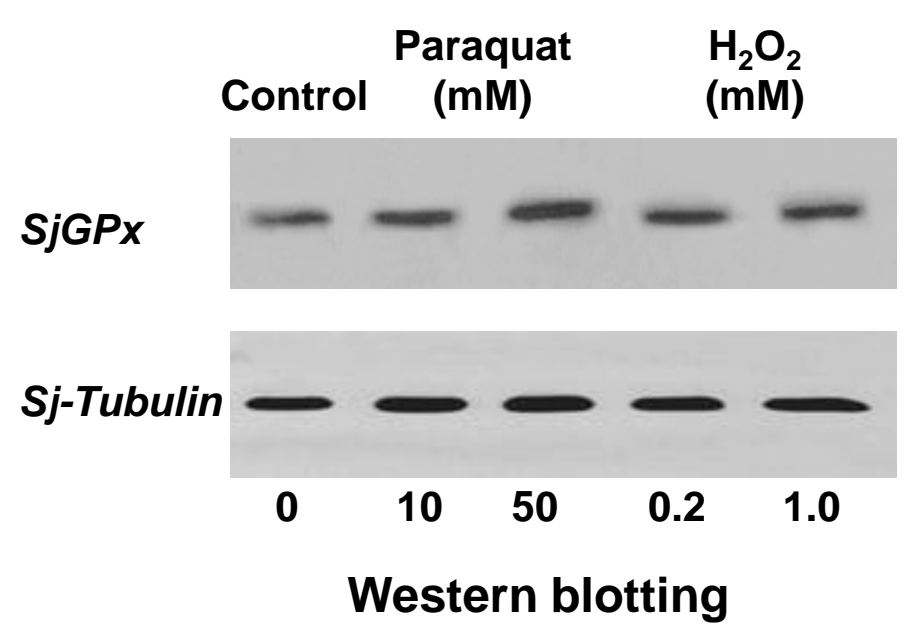

B

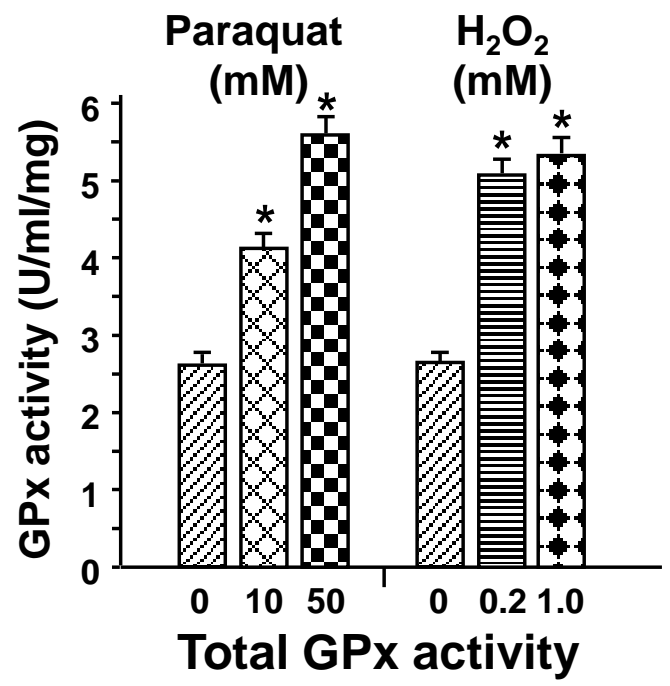




\section{Molecular cloning and characterization of a phospholipid hydroperoxide glutathione peroxidase gene from a blood fluke Schistosoma japonicum ${ }^{\text {th }}$}

Ying Zhanga ${ }^{a}$ Yuan He ${ }^{b}$, Li He ${ }^{b}$, Hong-Ying Zong ${ }^{b}$, Guo-Bin Caib, ${ }^{*}$

a Department of Medical Genetics, Wuhan University School of Basic Medicial Sciences, Wuhan, China

${ }^{\mathrm{b}}$ Department of Medical Parasitology, Wuhan University School of Basic Medicial Sciences, Wuhan, China

* Corresponding author at: Department of Medical Parasitology, Wuhan University School of Basic Medicial Sciences, Wuhan 430071, China.

Tel.: +862768759143.

E-mail address: gbcai2011@whu.edu.cn (G.B. Cai)

Summary: Glutathione peroxidase (GPx) in Schistosoma japonicum is seleniumdependent PHGPx, which might actively participate in the detoxification of oxidative damage during egg production. 


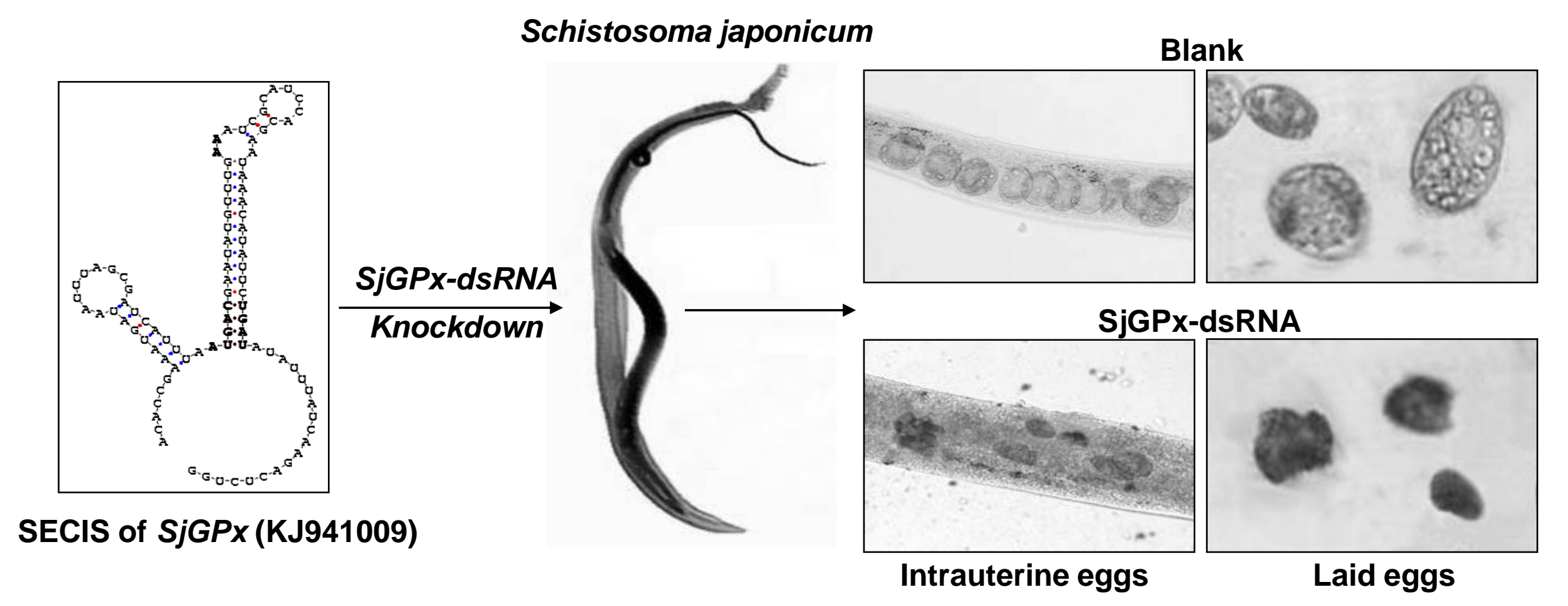

Zhang et al-Fig. of Graphical abstract 